11. Ramos JLA. Avaliação do crescimento intra-uterino por medidas antropométricas do recém-nascido [tese]. São Paulo: Faculdade de Medicina da Universidade de São Paulo; 1983.

12. Brenelli MA, Martins Filho J. Curvas de crescimento intrauterino da população de nascidos vivos na maternidade do CAISM - Unicamp. J Pediatr (Rio J) 1992;68:21-5.

13. Margotto PR. Curvas de crescimento intra-uterino estudo de 4413 recém-nascidos únicos de gestações normais. J Pediatr (Rio J) 1995;71:11-21.

14. Lopes JM. Crescimento fetal e neonatal. Por que não utilizar curvas nacionais? [Editorial] J Pediatr (Rio J) 1995;71:3-4.

15. Tavares RFS. Estudo do crescimento intra-uterino de recémnascidos normais. J Pediatr (Rio J) 1998;74:205-12.

16. Ott WJ, Doyle S. Normal ultrasonic fetal weight curves. Obstet Gynecol 1982;59:603.

17. Lubchenco LO, Hansman C, Dressler M, Boud E. Intrauterine growth as estimated from liveborn birth weight data at 24 to 42 weeks of gestation. Pediatrics 1963;32:793-800.
18. Tanner JM. Standards for birth weight on intrauterine growth (commentary). Pediatrics 1970;46:1-6.

19. Xiong X, Mayes D, Demianczuk N, Olson DM, Davidge ST, Newburn- Cook C, Saunders LD. Impact of pregnancy-induced hypertension on fetal growth. Am J Obstet Gynecol 1999;180:20713.

20. Sprauve ME, Lindsay MK, Drews-Botsch C, Graves W. Racial patterns in the effect of tabacco use on fetal growth. Am J Obstet Gynecol 1999;181:522-7.

21. Margotto PR. Crescimento intra-uterino. Percentis de peso, estatura e perímetro cefálico ao nascer de recém-nascidos únicos de gestações normais e seus correspondentes pesos placentários em diferentes períodos gestacionais [tese]. Montevideo, Uruguai: Centro Latinoamericano de Perinatologia y Desarrollo Humano (CLAP-OPS/OMS); 1992.

22. Goldenberg RL, Cutter GR, Hoffman HJ, Foster JM, Nelson KG, Hauth JC. Intrauterine growth retardation: Standards for diagnosis. Am J Obstet Gynecol 1989;161:271-7.

\title{
Asfixia neonatal e função tireoidiana
}

\author{
Neonatal asphyxia and thyroid function
}

\author{
Edna Maria de Albuquerque Diniz*
}

\begin{abstract}
A asfixia antes, durante ou após o nascimento é uma causa importante de mortalidade perinatal e morbidade neurológica. $\mathrm{O}$ conceito de asfixia perinatal permanece ainda controverso entre os vários autores. Acidose metabólica profunda ou acidose mista $(\mathrm{pH}<7,00$ no sangue da artéria umbilical), Apgar de 0 a 3 no $5^{\circ}$ minuto, sintomas e sinais no período neonatal imediato incluindo convulsões, hipotonia, coma ou encefalopatia hipóxica isquêmica (EHI), e evidência de disfunção de múltiplos orgãos são critérios aceitos pela maioria dos estudos para caracterizar a asfixia perinatal ${ }^{1,2}$.

Após um episódio asfíxico, algumas crianças podem se recuperar neurologicamente sem seqüelas, enquanto outras podem desenvolver déficits permanentes. Vários sistemas orgânicos podem ser afetados no recém-nascido (RN) de termo asfixiado, particularmente o sistema nervoso central (SNC) e os rins, que são os mais comprometidos ${ }^{4}$. Complicações cardiopulmonares são também descritas. A grande

* Professora Livre Docente de Neonatologia. Departamento de Pediatria da Faculdade de Medicina da Universidade de São Paulo (FMUSP). Chefe da Unidade de Cuidados Intensivos Neonatal do Instituto da Criança do Hospital das Clínicas da FMUSP.
\end{abstract}

maioria dessas lesões ocorre antes do nascimento, sendo primariamente resultante do comprometimento das trocas placentárias. Alteração do fluxo sangüíneo uterino, hipoxia materna, insuficiência placentária, prolapso e compressão do cordão umbilical podem interferir com a transferência de substratos para o feto ou alterar a passagem de catabólitos procedentes do feto para a sua mãe $e^{3,4}$.

No RN várias causas ainda podem levar à asfixia perinatal, entre elas trauma de parto, parto pélvico, síndromes aspirativas (síndrome de aspiração meconial, etc.), insuficiência respiratória de outras causas e crises de apnéia.

A asfixia, por sua vez, produz hipoxia, hipercapnia, acidose metabólica e insuficiência cardíaca, com redistribuição do fluxo sangüíneo para órgãos mais nobres como o $\mathrm{SNC}$, os rins e as adrenais, em detrimento de outros orgãos ${ }^{5}$. Alguns estudos têm demonstrado, também, alterações no funcionamento da glândula tireóide devido a episódios asfíxicos. Normalmente, logo após o nascimento de um RN de termo sadio, conforme descrito por Maciel $(1995)^{6} \mathrm{e}$ Knobel e Medeiros (1995) ${ }^{7}$,ocorre um aumento abrupto na liberação de TSH (hormônio estimulador da tireóide), atingindo cerca de $80-100 \mathrm{mU} / \mathrm{ml}$, o que leva à estimulação 
da captação de iodo e secreção dos hormônios tireoideanos pela glândula tireóide. De acordo com esses autores, a concentração sérica de TSH atinge seu valor maior após 30 minutos do nascimento, diminuindo rapidamente nas primeiras 24 horas de vida e daí mais lentamente. O T4 (tiroxina), por sua vez, atinge seu nível máximo 24 a 36 horas após o parto, declinando durante as primeiras duas semanas de vida. A concentração sérica de T3 aumenta em cerca de 5 a 6 vezes nas primeiras horas após o nascimento; em seguida, ocorre uma nova elevação do T3, diminuindo entre 36 e 48 horas de vida para valores de 150-250ng/dl. De acordo com Maciel ${ }^{6}$, a elevação inicial do T3 é devida à secreção tireoideana, enquanto a elevação posterior é conseqüente a um aumento da conversão periférica de $\mathrm{T} 4 \mathrm{a}$ T3. A concentração sérica do T3 reverso (R T3) permanece inalterada durante o período pós-natal imediato, diminuindo nas semanas seguintes. Os valores de T3 e T4 permanecem elevados durante a infância, diminuindo progressivamente até atingir, na adolescência, os valores considerados normais, de adultos. O RN prematuro reage de forma semelhante ao $\mathrm{RN}$ de termo em relação ao funcionamento da tireóide, porém quantitativamente em menor proporção, com valores séricos de TSH e de T3 mais baixos.

Vassart et al. (1998) ${ }^{8}$ referem que uma resposta inadequada à tirotropina pode levar a uma diminuição na atividade funcional da tireóide, ou seja, na síntese e secreção dos hormônios tireoideanos e no próprio crescimento da glândula. Neste aspecto, Wilson et al.(1982) ${ }^{9}$ determinaram as concentrações séricas de T4 livre por radioimunoensaio em 96 RN internados em UTI neonatal e em 32 RN saudáveis. Os autores constataram níveis mais baixos do hormônio naqueles RN portadores de insuficiência respiratória. Sugeriram que a medida do T4 livre poderá ser um bom parâmetro para a avaliação da função tireoideana. Franklin e O'Grady (1985) ${ }^{10}$ comentaram sobre a hipotiroxinemia transitória, constatada principalmente em $\mathrm{RN}$ prematuros portadores de insuficiência respiratória grave. Borges et al. $(1985)^{11}$, estudaram os vários aspectos da função tireoideana em três grupos de $\mathrm{RN}$ a termo: sete $\mathrm{RN}$ normais (grupo 1- controle), sete RN com Apgar transitoriamente baixo (grupo 2) e sete RN de mães com toxemia e hipertensão (grupo 3). As concentrações de TSH sérico, FT4 (tiroxina livre) e FT3 (tri-iodotironina livre) foram analisadas no sangue do cordão umbilical após cinco minutos do nascimento, e com 3, 24 e 48 horas após o parto. Os autores demonstraram que os níveis de TSH foram semelhantes nos três grupos logo após o parto. Porém o FT4 e a FT3 foram significativamente mais elevados no grupo controle que nos outros grupos durante as primeiras 48 horas de vida, embora no sangue de cordão os seus níveis tenham sido semelhantes nos três grupos. Os autores concluíram que as alterações na função tireoideana observadas nos RN asfixiados podem ser devidas ao menor consumo de $\mathrm{O}_{2}$ com taxa metabólica baixa, sugerindo ter a asfixia um papel importante no metabolismo da tireóide.
Nesta revista, uma pesquisa importante e atual foi desenvolvida por Pereira e Procianoy sobre os "efeitos da asfixia perinatal sobre os hormônios tireoideanos". Os autores estudaram $17 \mathrm{RN}$ a termo asfixiados e $17 \mathrm{RN}$ não asfixiados, nos quais foram dosadas as concentrações sangüíneas de T4, T3, FT4, T3 reverso e TSH no sangue do cordão e com 18 a 24 horas de vida. De forma semelhante ao que foi encontrado por outros autores ${ }^{10,11}$, não houve diferença significativa nos valores dos hormônios tireoideanos (com exceção do T3 reverso, que foi mais elevado no grupo asfixiado) no sangue do cordão umbilical entre os dois grupos de estudo. Entretanto, com 18 a 24 horas de vida, as concentrações séricas dos hormônios foram significativamente mais baixas no grupo de RN asfixiados em relação ao grupo controle, embora o rT3 fosse normal. Os autores concluíram que os níveis mais baixos obtidos dos hormônios eram secundários aos valores diminuídos de TSH, e os valores elevados e normais de $\mathrm{T} 3$ reverso refletiriam alteração no metabolismo periférico do T4. Este estudo demonstra os efeitos deletérios da asfixia perinatal sobre a tireóide, sugerindo a necessidade crescente da prevenção da asfixia.

\section{Referências bibliográficas}

1. Carter BS, Haverkamp AD, Haverkamp AD, Merenstein GB. The definition of acute asphyxia. Clin Perinatol 1993;20(2):287304.

2. Williams CE, Mallard C, Tan W, Gluckman PD. Pathophysiology of perinatal asphyxia. Clin Perinat 1993;20(2):305-26.

3. Hill A, Volpe JJ. Perinatal asphyxia: clinical aspects. Clin Perinatol 1989;16(2):435-57.

4. Perlman JM. Systemic abnormalities in term infants following perinatal asphyxia relevance to long-term neurologic outcome. Clin Perinatol 1989;16(2):475-84.

5. Pereira DN, Procianoy RS. Transient elevation of aldosterone levels in perinatal asphyxia. Acta Paediatr 1997;86:851-3.

6. Maciel RMB. Interpretação laboratorial nas doenças da tireóide. In: Setian N, ed. Endocronologia Pediátrica. Aspectos físicos e metabólicos do RN ao adolescente. São Paulo: Sarvier; 1989. p. 253-62.

7. Knobel M, Medeiros Neto, GA. Embriologia da glândula tireóide. In: Setian N, ed. Endocronologia Pediátrica. Aspectos físicos e metabólicos do RN ao adolescente. São Paulo: Sarvier; 1989. p. 213-43.

8. Vassart G, Dumont JE, Refetoff S. Thyroid disorders. In: Scriver CR, Beaudet AL, Sly WS, Valle D. The Metabolic and Molecular Bases of Inherited disease. New York: McGraw-Hill; 1995. p. 2883-903.

9. Wilson DM, Hopper AO, McDougall IR, Bayer MF, Hintz RL, Stevenson DK, et al. Serum free thyroxine values in term, premature, and sick infants. J Pediatr 1982;101(1):113-7.

10. Franklin R, O'Grady C. Neonatal thyroid function: Effects of nonthyroidal illness. J Pediatr 1985;107(4):599-602.

11. Borges M, Lanes R, Moret LA, Balochi D, Gonzalez S. Effect of asphyxia on free thyroid hormone levels in full term newborns. Pediatr Res 1985;19(12):1305-7. 